\title{
The Problem of Identifying Possible Signals of Extra-Terrestrial Civilizations in the Framework of the Information-Based Method
}

\author{
Boris Menin \\ Refrigeration Consultancy Ltd., Beer-Sheba, Israel \\ Email: meninbm@gmail.com
}

How to cite this paper: Menin, B. (2019) The Problem of Identifying Possible Signals of Extra-Terrestrial Civilizations in the Framework of the Information-Based Method. Journal of Applied Mathematics and Physics, 7, 2157-2168.

https://doi.org/10.4236/jamp.2019.710148

Received: August 27, 2019

Accepted: October 5, 2019

Published: October 8, 2019

Copyright $\odot 2019$ by author(s) and Scientific Research Publishing Inc. This work is licensed under the Creative Commons Attribution International License (CC BY 4.0).

http://creativecommons.org/licenses/by/4.0/

\begin{abstract}
Aims: The purpose of this work is to formulate the requirements for future methods of searching for extra-terrestrial civilizations by use of the concepts of information theory and the theoretically grounded method. Methodology: To realize it, the number of dimensionless criteria contained in the International System of Units (SI) has been calculated. This value, without additional assumptions, allows us to present a formula for calculating the comparative uncertainty of the model of any physical phenomenon. Based on these formulas, the magnitude of the inevitable threshold of misunderstanding of two civilizations in the universe is determined. Results: New theoretical recommendations for choosing the most effective methods to search the techno signatures of extra-terrestrial civilizations are formulated. Conclusion: Using the calculated amount of information embedded in the model, we showed that the most promising methods for finding potential residents in the Universe should combine frequency radiation with thermal or electromagnetic quantities.
\end{abstract}

\section{Keywords}

Extra-Terrestrial Intelligence, Amount of Information, SETI, SI, Technosignature, Uncertainty

\section{Introduction}

The amazing idea of the existence of extra-terrestrial civilizations has its supporters and opponents, numerous on each side. The first theoretical studies on this topic to be published in the scientific literature, apparently were presented in the early sixties of the twentieth century. Because the direct study of the vicin- 
ity of stars using automatic and habitable spacecraft is obviously impossible, scientists are forced to consider only remote sensing.

Sixty years ago, Cocconi and Morrison suggested choosing a $21.0 \mathrm{~cm}$ wave because it should be known to all civilizations as the emission of neutral interstellar hydrogen. Therefore, scientists assume that on this wave there is a significant probability of accidental detection of artificial radiation. At the same time, the search for intelligent life in the Universe was based on the assumption that there is a mutual desire of extra-terrestrial civilizations to find each other.

Debates on the probability of finding intelligent life are still ongoing. Back in 1961, astronomer Drake proposed [1] a formula that estimates the number of potentially intellectual civilizations in the galaxy. Most of the variables in the equation are still estimates of uncertainties. The opposite opinion is based on the theory of the Italian physicist Enrico Fermi and states that if another intellectual form of life really existed, we would definitely have intersected with it by now.

With the development of scientific and technological progress and the emergence of powerful radio telescopes such as Kepler and Transiting Exoplanet Survey Satellite (TESS) in Earth orbit, it became possible to thoroughly research space, search SETI (search for extra-terrestrial intelligence) and tech nosignatures. The latter term means radio emission, optical and infrared radiation, and laser radiation and signals from a massive structure or a polluted atmosphere, which are signs of advanced intelligence [2]. At the same time, the latest report from SETI declares that the team did not find anything this time around [3].

In general, unfortunately, the projects of special search for signals, in which hundreds of millions of American dollars were invested, did not give positive results. Currently, there is no evidence of the existence of extra-terrestrial civilizations above the threshold of observational capabilities achieved by our civilization.

From this we can draw the following conclusions (although the author is convinced that readers will find other, directly opposite conclusions):

- the generally accepted theory(ies) of the search for extra-terrestrial civilizations is incorrect;

- observational data are insufficient to draw any conclusions;

- human civilization is unique, and the theory is correct, but there are no extra-terrestrial civilizations.

Of course, there may be other claims, for example, civilizations, having reached the technological phase, quickly die, for example, from environmental pollution, nuclear war, etc., without having time to solve communication problems with other civilizations. The author provides readers with the opportunity to come up with any guesses and suggestions.

The purpose of this article is proposed to consider the problem of choosing methods for searching extra-terrestrial civilizations from the point of view of information theory, which, from the author's point of view, is universal: only practical activity and information exchange bring us into contact with the mate- 
rial world and allow us to generate scientific knowledge. The same can be said about the possible life outside the Earth. This position is clearly absent in most search patterns of the mind in the universe.

\section{Informational Measure of the Model's Inconsistency}

When formulating new methods for detecting possible signals or manifestations of intelligent activity of extra-terrestrial civilizations, scientists try to describe the observed phenomena with the help of concepts instilled in human everyday experience, acquired knowledge and, often, intuition.

At the same time, despite the 100-year-old efforts, it has not yet been possible to create a unified theory uniting classical determinism, general theory of relativity and probabilistic laws of quantum mechanics. So far, the only tool that unites all modern physics is that scientists use the System of Base Quantities (SBQ), such as the International System of Units (SI), to implement their ideas. This means that the harmonious construction of modern science is based on the simple agreement that any physical laws of micro- and macrophysics from the point of view of mankind are described by fairly specific dimensional quantities: base and derived quantities. Accepting a quantity as a fundamental means that it can be assigned as a measurement standard, which does not depend on the standard chosen for another fundamental quantity. Base quantities are chosen arbitrarily, while derived quantities are chosen to satisfy the detected physical laws or the corresponding definitions.

The concept of SBQ is taken from our everyday experience and is valid only for short-term perception by human beings of observed phenomena. It would be surprising if someday it was possible to exclude concepts from the physical theory, which are the very basis of our daily life. True, the history of science reveals the amazing fruitfulness of human thought, and one should not lose hope. However, until we succeed in spreading our ideas in this direction, we must, with greater or less difficulty, squeeze the observed phenomena into the framework of the concept of SBQ. Although we will always be bothered by the feeling that we are trying to insert a huge human foot into a small diamond shoe that does not suit her.

$\mathrm{SBQ}$, in its essence, is a new element of scientific knowledge, completely alien to classical concepts. It exists only due to the consensus of researchers, although there is no SBQ in nature. By default, the use of dimensional quantities contained in an SBQ to describe the micro- and macrocosm implies a certain structure that limits our knowledge. Thus, quantities/variables are selected in a pre-agreed $S B Q$, which is a set of dimensional quantities that are base and generated derived quantities. These quantities are necessary and sufficient to describe the known laws of nature [4]. This means that any scientific knowledge and, without exception, all formulated physical laws are discovered thanks to the information contained in the SBQ. This is a unique channel (generalizing information carrier [5]) through which information is transmitted to the observer or the observer retrieves information about the object from the SBQ. The SBQ in- 
cludes a finite number of physical dimensional quantities that can characterize the physical properties of the world and, in particular, the observed phenomenon qualitatively and quantitatively. So, the observation of a material object and its modelling are limited to the scope of SBQ. We model only what we can imagine or observe, and the mere presence of a selected SBQ, such as a lens, sets a certain limit for measuring an observed object.

Each quantity carries a certain amount of information about the object being studied. Because the number of elements in SBQ is finite (see below), the total amount of information contained in SBQ is, of course, finite too. Thus, we conclude that there is a certain limit to knowledge about the surrounding reality. This limit is not due to any existing physical laws but to the presence of collective human consciousness. The very fact of the measurement process presupposes the existence of an already formulated physical and mathematical model of the object under study, including equations and boundary conditions. In this case, it is already possible to make a list of the registered dimensional quantities and calculate their number in advance. Most importantly, this is the ability to calculate the change in entropy between the initial state corresponding to the maximum number of quantities in the SBQ and the final one corresponding to a certain number of quantities in the model.

The SBQ includes base and derived quantities used to describe various classes of phenomena $(\mathrm{CoP})$. In other words, the additional limits of the description of the material object under study are determined by the choice of the CoP and the number of derived quantities taken into account in the mathematical model [6]. For example, in mechanics, SI uses the basis $\{L$-length, $M$-mass, $T$-time $\}$, i.e. $\mathrm{CoP}_{\mathrm{SI}} \equiv L M T$. There are four additional base quantities: $\Theta$ is a thermodynamic temperature, $I$ is the amount of electric current, $J$ is luminous intensity and $F$ is the amount of substance.

If the SBQ and CoP are not specified, the definition of 'information about the object under study' loses its force. Without SBQ, modelling a phenomenon is impossible. 'You can never get something out of nothing, even by watching' [7]. $\mathrm{SBQ}$ can be interpreted as the basis of all the available knowledge that people can have about the environment at present.

SBQ has a function called universality, which stems from a deep connection with mathematics and information theory, and helps to model complex systems involving any physical phenomenon. With a certain degree of imagination, we can assume a situation where a material object has a huge number of properties (criteria) that characterize its content and interaction with the environment. Then, we assume that each dimensionless complex can provide the observer with some information about the dimensionless field under investigation $u$ (the observed object). In other words, a researcher studying a physical phenomenon chooses-in accordance with his experience, knowledge and intuition-certain characteristics of the object. With this choice of an object, the links between the real object and its environment are destroyed. In addition, the model designer takes into account a relatively smaller number of quantities, compared with the 
existing reality, due to lack of time, as well as technical and financial resources. Therefore, the 'image' of the investigated object is displayed in the model with a certain uncertainty, which depends primarily on the number of quantities taken into account. In addition, different groups of researchers who use different approaches to solve specific problems and, accordingly, different groups of quantities that differ from each other in quality and quantity, can access the object. Thus, for any physical problem, the appearance of a certain quantity in the model can be considered as a random process.

So, it is assumed that taking the quantity/criteria into account by the researcher is equally probable. When researching an object at the stage of model development, it is advisable to use vector (positional) methods for presenting information about the observed phenomena. When there are a large number of components (large vector space), only two states of the vector component can be distinguished: for example, the presence or absence of a signal; in our case, the appearance or absence of the specific quantity taken into account. It should be noted that the statement of the equally probable appearance of the quantities is justified by the aim of the study: finding the absolute uncertainty $\Delta_{p m m}$ due to the level of detail of the object under study. Indeed, any other distribution of readings (quantities) gives less information, which leads to a larger model's uncertainty compared with the uncertainty calculated with a uniform distribution of quantities.

This approach is universal in the study of any physical phenomenon. It is believed that universality occurs when a system is very complex, consisting of many parts that interact strongly with each other. Universality is very common in nature. Wigner's hypothesis states [8] that all complex, correlated systems demonstrate universality, from the crystal ice to the Internet. The more complex the system, the more reliable its versatility should be.

At the same time, the uncertainty of a mathematical model with a finite number of quantities cannot be infinitely small. This is because this uncertainty is related to the reliability of each natural or computer experiment and should be part of the theory of measurement. When this theory is used as a physical model, it becomes the object of application of both of the above limitations. In physics, this leads to the assumption about the possibility of the existence of certain uncertainty (limited accuracy) before applying the mathematical model.

There are fundamental objective (for example, thermodynamic) limits of accuracy when conducting an experimental study. This, in turn, determines the presence of an a priori source of inaccurate knowledge of all material objects, information about which is received and processed by the observer.

The fundamental limitations of the maximum accuracy with which we can determine physical quantities at present are determined by the Heisenberg uncertainty principle [9]. However, the Planck constant is extremely small, so this uncertainty in the macro world is devoid of practical significance. The uncertainties of position and momentum resulting from this principle go far beyond the limits of achievable accuracy of experiments. 
In turn, the creation of a certain SBQ (in our case, SI) means that we are talking about trying to limit the set of possible quantities to a smaller number of base quantities. Then, all other required quantities can be found or determined based on these base quantities, which must meet certain criteria [4].

\subsection{Initial Unremovable Blur of the Object of Study}

The hypotheses proposed so far about the existence of intelligent life in the universe remain highly speculative and rely in large part only on the assumption of some universal motives or social dynamics of aliens, although many scientists argue that there is a possibility that we are alone [10].

Continuing our reasoning, it should be noted that any model contains a certain amount of information that allows you to choose the optimal number of quantities taken into account. The following is a simplified short introduction to the above statements [11].

Obviously, the researcher must develop a model that takes into account certain features of the signals of an extra-terrestrial civilization, including any optical, frequency in a certain range, electrical or thermodynamic parameters. In this case, the scientist uses quantities of the SI, which includes both base and derived quantities, on the basis of which various CoPs are built. The dimension of the derived quantity $q$ can be introduced by the following equation [6] [12]

$$
q \ni L^{l} \cdot M^{m} \cdot T^{t} \cdot I^{i} \cdot \Theta^{\Theta} \cdot J^{j} \cdot F^{f}
$$

where $l, m, \cdots, f$ are the exponents of base quantities, which are limited to the following intervals [13]:

$$
\begin{aligned}
& -3 \leq l \leq+3, \quad-1 \leq m \leq+1, \quad-4 \leq t \leq+4, \quad-2 \leq i \leq+2, \\
& -4 \leq \theta \leq+4, \quad-1 \leq j \leq+1,-1 \leq f \leq+1 .
\end{aligned}
$$

The number of options for the dimensions of the exponents of base quantities $e_{l}, e_{m}, \cdots, e_{f}$ are

$$
e_{l}=7 ; e_{m}=3 ; e_{t}=9 ; e_{i}=5 ; e_{\theta}=9 ; e_{j}=3 ; e_{f}=3
$$

So, SI contains the total number of dimensionless complexes $\mu_{\mathrm{SI}}$

$$
\mu_{\mathrm{SI}}=\left(e_{l} \cdot e_{m} \cdot e_{t} \cdot e_{i} \cdot e_{\theta} \cdot e_{j} \cdot e_{f}-1\right) / 2-7=38.265
$$

where -1 corresponds to the case when $e_{l}, e_{m}, \cdots, e_{f}$ are equal to zero in Equation (1). The SI includes direct and inverse quantities, for example, length $\left(L^{1}\right)$ and running length $\left(L^{-1}\right)$. Because an amount of information about an object having symmetrical parts can be calculated from its one part, while others are considered informationally empty, the total number of quantities can be divided by 2 . As already noted, the SI contains seven-7-basic quantities.

It can be stated that the proposed method can be applied to any models in the search for activity of extra-terrestrial civilizations. It is explained by the fact that the relative and comparative uncertainties of the dimensional quantity $U$ and the dimensionless quantity $u$ are equal:

$$
\Delta U / S^{*}=(\Delta U / a) /\left(S^{*} / a\right)=\Delta u / S
$$




$$
(r / R)=(\Delta U / U) /(\Delta u / u)=(\Delta U / U) \cdot(a / \Delta U) \cdot(U / a)=1,
$$

where $S$ is the dimensionless interval of changes and $\Delta u$ is the dimensionless overall absolute uncertainty when measuring the dimensionless squantity $u ; S^{*}$ is the dimensional interval of changes and $\Delta U$ is the dimensional overall absolute uncertainty when measuring the dimensional quantity $U$; $a$ is a scaling factor; $r$ is the relative uncertainty of the dimensional quantity $U$; and $R$ is the relative uncertainty of the dimensionless squantity $u$. Equation (5) proves the possibility of applying the information method for both dimensional and dimensionless quantities.

Based on the above formulae, it is possible to formulate the necessary condition for choosing the optimal version of the model that describes the phenomenon under study, in particular, the process of searching for a signal from an extra-terrestrial civilization. Namely [11]: when modelling a physical phenomenon, a system of base quantities is specified, in which there are $\Psi$ dimensional quantities and $\xi$ base quantities. The developer chooses the target dimensionless basic quantity $u$, the values of which are located in the interval $S$, and specifies the class of phenomena with the total number of dimensional quantities $z^{\prime}$ and the number of base quantities $\beta^{\prime}$. In this case, for a given number of dimensional quantities $z^{\prime}$ and a selected number of base quantities $\beta^{\prime}$ in a model, the absolute uncertainty $\Delta_{p m m}$ when measuring $u$ is determined by the relation:

$$
\Delta_{p m m}=S \cdot\left[\left(z^{\prime}-\beta^{\prime}\right) / \mu_{\mathrm{SI}}\left(z^{\prime \prime}-\beta^{\prime \prime}\right) /\left(z^{\prime}-\beta^{\prime}\right)\right]
$$

where $\varepsilon=\Delta_{p m m} / S$ is comparative uncertainty [7].

Equation (6), surprisingly, turns out to be very simple. Absolute and relative uncertainties are familiar to physicists. As for the comparative uncertainty, it is rarely mentioned. Nevertheless, comparative uncertainty is of great importance for the application of information theory in physics and engineering sciences [7].

Equation (6) has a physical meaning. The $\mu_{\mathrm{SI}}$ hypothesis states that in nature there is a fundamental limit to the accuracy of measurement of any process that cannot be surpassed by any improvement in the tools, measurement methods or computerization of the model. The value of this limit is much higher and stronger than the Heisenberg uncertainty relation dictates and imposes additional serious restrictions on the search and detection of reasonable activity in the universe.

At its core, $\Delta_{p m m}$ is an a priori conceptual uncertainty that is inherent in any physical and mathematical model and is independent of the measurement process. This is only due to the decision of the developer to choose a specific class of phenomena and the number of quantities used to describe the observed signal. Moreover, the general (integral) uncertainty of the model, including the uncertainties due to the simplification of the original object or phenomenon, as well as the uncertainties caused by the transition from the mathematical model to the numerical method, will be much larger than $\Delta_{p m m}$. Thus, the inaccurate (fuzzy) representation of an object depends both on the class of phenomena and on the number of quantities selected and taken into account by the conscious observer; 
the latter directly depends on the knowledge, life experience and intuition of the researcher.

Equating the derivative of $\Delta_{p m m} / S$ (6) with respect to $z^{\prime}-\beta^{\prime}$ to zero, we obtain the following condition for achieving the minimum comparative uncertainty for a particular $\mathrm{CoP}_{\mathrm{sI}}$ :

$$
\left(z^{\prime}-\beta^{\prime}\right)^{2} / \mu_{\mathrm{SI}}=\left(z^{\prime \prime}-\beta^{\prime \prime}\right) .
$$

Using (7), one can find the values for the lowest attainable comparative uncertainty for different $\mathrm{CoP}_{\mathrm{SI}}$; it should be noted that the values of the comparative uncertainties and the recommended number of selected quantities are different for each $\mathrm{CoP}_{\text {sI: }}$

when searching for a certain frequency $\left(\mathrm{Hz}=\mathrm{c}^{-1}\right)$ in a given range $\left(\mathrm{CoP}_{\mathrm{SI}} \equiv T\right)$, taking into account the above explanations, the smallest comparative uncertainty of $\varepsilon_{T}$ can be achieved under the following conditions:

$$
\begin{aligned}
& \left(z^{\prime}-\beta^{\prime}\right)=\left(e_{t}-1\right) / 2-1=(9-1) / 2-1=3 \\
& \left(z^{\prime \prime}-\beta^{\prime \prime}\right)=\left(z^{\prime}-\beta^{\prime}\right)^{2} / \mu_{C И}=9^{2} / 38.265=0.0021<1
\end{aligned}
$$

where -1 corresponds to the case when all exponents of the main quantities are zero in formula (1); division by 2 indicates the presence of direct and inverse values, for example, $L^{1}$ is the length, $L^{-1}$ is the running length; and 1 corresponds to one basic quantity $T$.

According to (7) and (8), $\boldsymbol{\varepsilon}_{T}$ is equal to:

$$
\varepsilon_{T}=(\Delta u / S)_{T}=3 / 38.265+0.0021 / 3=0.000078 .
$$

In other words, according to (8), even one dimensionless basic quantity does not allow achieving the smallest comparative uncertainty. Consequently, within the framework of the proposed approach, the initial comparative uncertainty cannot be realized using any model with one, two base quantities (for example, $\left.\mathrm{CoP}_{\mathrm{SI}} \equiv T, \mathrm{CoP}_{\mathrm{SI}} \equiv L T, \mathrm{CoP}_{\mathrm{SI}} \equiv M T\right)$ or mechanistic model $\left(\mathrm{CoP}_{\mathrm{SI}} \equiv L M T\right)$. Moreover, the greater the number of mechanical parameters, the greater the inherent uncertainty. In other words, no frequency radiation or optical methods, in the framework of the information-oriented approach, are recommended for a search for signals from extra-terrestrial civilizations. This statement is confirmed by the data of Table 1 on comparative uncertainties and recommended number of dimensionless criteria in models for several CoP:

The reader must remember that the optimal number of criteria selected in the model corresponds to the comparative uncertainty inherent in a particular $\mathrm{CoP}_{\mathrm{sI}}$. Because the values of comparative uncertainty and the required number of selected quantities are completely independent and different for each class of phenomena, the proposed approach is a universal metric for comparing different models describing the same object of study.

\subsection{Maintaining Insurmountable Barrier in Understanding an Alien Civilization}

As already noted in Chapter 2, the only tool that is used in all areas of human 
Table 1. Comparative uncertainties and recommended number of dimensionless criteria.

\begin{tabular}{ccc}
\hline CoPsi $_{\text {si }}$ & Comparative uncertainty & Optimal number of criteria \\
\hline$T$ & 0.000078 & $0.0021<1$ \\
$M T$ & 0.00058 & $0.0032<1$ \\
$L T$ & 0.0015 & $0.022<1$ \\
$L M T$ & 0.0048 & $0.2<1$ \\
$L M T F$ & 0.0146 & $\cong 2$ \\
$L M T I$ & 0.0245 & $\cong 6$ \\
$L M T \theta$ & 0.0442 & $\cong 19$ \\
\hline
\end{tabular}

activity is $\mathrm{SBQ}$ (SI). It reflects the long-term knowledge accumulated over 12,000 years of community development. Therefore, a common indicator of the vastness of our knowledge is the value of $\mu_{\mathrm{SI}}$. An alien civilization must have an idea of their world, which would reflect to their knowledge systems, like ours. We denote this indicator $\mu_{\mathrm{ET}}$.

Let us try to compare these two values to find out, of course, very relatively, but on the basis of a theoretically sound information method, the depth of the abyss or the approximate correspondence of what we have with the volume of alien scientific treasures. The author, not being a zealous optimist or avid pessimist, admits the possibility that in distant worlds rational beings do not have nine senses (sight, hearing, taste, smell, touch, a feeling of warmth (or lack thereof) on our skin, a sense of balance, which determined by the fluid-containing cavities in our inner ear, the perception of pain by the skin, joints and organs of the body and "body awareness"- understanding where our body parts are, even if we do not feel or see them) and intuition. It is these feelings, along with our activity in the surrounding material world and the exchange of information that allowed us to rise to the highest level in terms of science, technology, biology and psychology. Unfortunately, we do not know what is on the other side of the potential contact. While we can only expect a miracle or step by step to understand nature and another's mind.

Continuing our reasoning, one can note that the model of the investigated object in the observed universe contains a certain amount of information $\Delta B_{e S I}$ calculated using the formula [14]

$$
\Delta B_{\mathrm{eSI}}=\kappa \cdot \ln \left[\mu_{\mathrm{SI}} /\left(z^{\prime \prime}-\beta^{\prime \prime}\right)\right]
$$

where $\Delta B_{\mathrm{eSI}}$ is expressed in units of entropy [14], $z^{\prime}$ and $\beta^{\prime}$ are, respectively, the number of all and base quantities registered in a model of the signal from a specific object, $\kappa$ is the Boltzmann constant.

To calculate the amount of information in bits $\Delta B_{\text {bSI }}$ embedded in a model, it is necessary to divide $\Delta B_{\text {eSI }}$ by $k \ln 2=9.569926 \times 10^{-24} \mathrm{~kg} \cdot \mathrm{m}^{2} \cdot \mathrm{s}^{-2} \cdot \mathrm{K}^{-1}$ [7] [14]:

$$
\Delta B_{\mathrm{bSI}}=\ln \left[\mu_{\mathrm{SI}} /\left(z^{\prime \prime}-\beta^{\prime \prime}\right)\right] / \ln 2(\text { bits })
$$

Proceeding from (11) and comparing different models proposed by two dis- 
tant intelligent civilizations and which study the same technological process/physical phenomenon/ signal/techno signatures, you can determine the significance of the inevitable threshold misunderstanding of neighbours $\Delta B_{612}$ in the universe:

$$
\begin{aligned}
\Delta B_{\mathrm{b} 12} & =\ln \left[\mu_{\mathrm{SI}} /\left(z_{1}^{\prime \prime}-\beta_{1}^{\prime \prime}\right)\right] / \ln 2-\ln \left[\mu_{\mathrm{ET}} /\left(z_{2}^{\prime \prime}-\beta_{2}^{\prime \prime}\right)\right] / \ln 2 \\
& =\left(\ln \mu_{\mathrm{SI}}-\ln \left(z_{1}^{\prime \prime}-\beta_{1}^{\prime \prime}\right)-\ln \mu_{\mathrm{ET}}+\ln \left(z_{2}^{\prime \prime}-\beta_{2}^{\prime \prime}\right)\right) / \ln 2 \\
& =\left\{\ln \left(\mu_{\mathrm{SI}} / \mu_{\mathrm{ET}}\right)-\ln \left[\left(z_{1}^{\prime \prime}-\beta_{1}^{\prime \prime}\right) /\left(z_{2}^{\prime \prime}-\beta_{2}^{\prime \prime}\right)\right]\right\} / \ln 2 \text { (bits) }
\end{aligned}
$$

Where $z_{1}^{\prime}$ and $\beta_{1}^{\prime}$ are, respectively, the number of quantities and the number of the base quantities in a model of the first civilization; $z^{\prime}$ and $\beta_{2}^{\prime}$ are, respectively, the number of quantities and the number of the base quantities recorded in a model of the second civilization.

If $\Delta B_{612}<0$, then a model of the second civilization contains more detailed information about the observed phenomenon. If $\Delta B_{b 12}>0$, then a model of the first civilization is more informative. It is obvious that the $\Delta B_{612}$ cannot be equal to zero due to the low probability of the situation when $\mu_{\mathrm{SI}}=\mu_{\mathrm{ET}}$ and $z_{1}^{\prime}-\beta_{1}^{\prime}=z_{2}^{\prime}-\beta_{2}^{\prime}$.

Even if we assume that $z_{1}^{\prime}-\beta_{1}^{\prime}=z_{2}^{\prime}-\beta_{2}^{\prime}$, due to the aforementioned characteristics of humanity, $\Delta B_{b 12}$ will not be zero, and this will significantly reduce the success of a possible meaningful contact between two civilizations. Consequently, information about the rationality of life on Earth sent by the Voyager spacecraft (sounds and images chosen to represent the diversity of life and culture on Earth) is unlikely to be decoded by aliens. This situation greatly complicates the search for convincing signals of extra-terrestrial life, due to the presence of unavoidable blurriness of perceived signals, which results in a non-zero $\Delta B_{612}$ value. Thus, we have problems with the reception of extra-terrestrial transmissions. On the other hand, even if we accept such a transmission, it may be that it will not be perceived as a reasonable signal.

It should be noted that formula (12) was obtained without any assumptions. Therefore, the proposed approach makes it much easier to interpret the results achieved compared with the Drake formula or Kardashev's classification of extra-terrestrial civilizations in accordance with the energy that they have [15]. These formulae, proposed dozens of years ago, contain variables, the exact values of which are unknown. The possible limits of their changes are so huge that they lead to results that differ hundreds and thousands of times.

\section{Conclusions}

This article presents an information method and its possible applications for the analysis of methods for finding an extra-terrestrial civilization. It is theoretically justified and, surprisingly simple in perception and explanation of the results obtained. With its conclusion, there are no assumptions, and it is deprived of the subjectivity of the researcher who makes decisions in accordance with his knowledge, experience and intuition. The presented formula (6) for calculating 
the absolute and comparative uncertainties of the model designed for searching techno signatures is simple in form and does not require much effort on the part of the developer to apply it.

Comparative uncertainty is a theoretically sound tool that allows scientific groups to provide substantial evidence in favour of the identified relationship or effect obtained in the course of research. The present study suggests that ignoring comparative uncertainty leads to a situation where scientific statements are far from achieving real results, positive or negative.

Thus, the new theoretical positions can be formulated:

- System of Base Quantities (SBQ, in our case, SI) can be interpreted as the basis of all the available knowledge that people can have about the environment at present,

- in nature there is a fundamental limit to the accuracy of measurement of any process that cannot be surpassed by any improvement in tools, measurement methods, or computerization of a model;

- any frequency radiation or optical methods, in the framework of the information-oriented approach, is not recommended for a search of signals from extra-terrestrial civilizations;

- the presence of unavoidable blurriness of perceived signals, results in a non-zero $\Delta B_{b 12}$ value.

But even if we are not able to use the frequency of radiation to search for potential candidates of existing civilizations, what are our chances to observe them? We must select methods for finding potential residents combining thermal $\left(\mathrm{CoP}_{\mathrm{SI}} \equiv L M T \theta\right)$ or electromagnetic quantities $\left(\mathrm{CoP}_{\mathrm{SI}} \equiv L M T I\right)$. In this case, the magnitude of the experimentally comparative uncertainty, using high-precision measuring instruments, can be close to the theoretically achievable. However, this limit means that in any case, the perceived signals will be "blurred" for observation.

Despite huge investments, the use of super-powerful computers, unique algorithms and high-precision measuring equipment, the continued use of only frequency search methods to detect extra-terrestrial civilizations without taking into account electromagnetic or thermodynamic components in observation models, unfortunately, is doomed to failure. There is no thorough search programme, despite the fact that SETI of NASA created the necessary base for identifying a developed extra-terrestrial civilization. Unlike other areas of physics and technology, its theoretically based search has become static. We will probably find the answer whether our Earth is unique or not, but an analysis of history shows that it is unlikely to be able to find out in the foreseeable future.

If the results of the presented work are positively received, they will help theorists to choose the most suitable models and methods that have comparative uncertainties that are the closest to the theoretically recommended ones. This will help increase the likelihood of detecting extra-terrestrial civilizations if they exist. However, at the moment, it should be recognized that the emergence of human life is a very rare combination of subtle states. And the appearance of intel- 
ligent life is an extremely rare event. Perhaps the only one in our galaxy.

\section{Conflicts of Interest}

The author declares no conflicts of interest regarding the publication of this paper.

\section{References}

[1] Lamb, D. (2005) The Search for Extra-Terrestrial Intelligence: A Philosophical Inquiry. Routledge, London and New York, 1-222.

https://doi.org/10.4324/9780203991749

[2] Wilson, T.L. (2001) The Search for Extra-Terrestrial Intelligence. Nature, 409, 1110-1114. http://sci-hub.tw/10.1038/35059235 https://doi.org/10.1038/35059235

[3] Price, D.C. (2019) The Breakthrough Listen Search for Intelligent Life: Observations of 1327 Nearby Stars over 1.10-3.45 GHz. 1-19. http://seti.berkeley.edu/listen2019/BL1327stars.pdf

[4] Sonin, A.A. (2001) The Physical Basis of Dimensional Analysis. 2nd Edition, Department of Mechanical Engineering, MIT, Cambridge. http://goo.gl/2BaQM6

[5] Burgin, M. (2010) Theory of Information: Fundamentality, Diversity and Unification. World Scientific Publishing, Singapore. https://doi.org/10.1142/7048

[6] Sedov, L.I. (1993) Similarity and Dimensional Methods in Mechanics. 10th Edition, CRC Press, Boca Raton.

https://www.scribd.com/document/407227296/Preview-Of-Similarity-and-dimensi onal-methods-pdf

[7] Brillouin, L. (2004) Science and Information Theory. Dover, New York.

[8] Irwin, K. (2018) Toward the Unification of Physics and Number Theory. 1-55. https://pdfs.semanticscholar.org/a1c8/0f39d75a2fab44d35a8d53f2df98eef215d8.pdf

[9] Heisenberg, W. (1927) Uber den anschaulichen Inhalt der quantentheoretischen Kinematik und Mechanik. Zeitschrift für Physik, 43, 172-198. https://doi.org/10.1007/BF01397280

[10] Sandberg, A., Drexler, E. and Ord, T. (2019) Dissolving the Fermi Paradox. 1-19. https://arxiv.org/pdf/1806.02404.pdf

[11] Menin, B. (2017) Information Measure Approach for Calculating Model Uncertainty of Physical Phenomena. American Journal of Computational and Applied Mathematics, 7, 11-24. https://goo.gl/m3ukQi

[12] Bich, W. (2019) The Third-Millennium International System of Units. Rivista Del Nuovo Cimento, 42, 49-102. http://www.roma1.infn.it/ luci/LabSS/new SI system.pdf

[13] NIST (2008) Special Publication 330 (SP330) the International System of Units (SI). https://www.nist.gov/sites/default/files/documents/2016/12/07/sp330.pdf

[14] Menin, B. (2015) Possible Limits of Accuracy in Measurement of Fundamental Physical Constants. International Refereed Journal of Engineering and Science, 4, 8-14. http://goo.gl/HjYBOs

[15] Kardashev, N.S. (1964) Transmission of Information by Extra-Terrestrial Civilizations. Soviet Astronomy, 8, 217-221. 